\title{
Relationship Between Blood Pressure Levels on Admission and the Onset of Acute Pneumonia in Elderly Patients With Cerebral Hemorrhage
}

\author{
Taishi Ishisaka ${ }^{a}$, Yuta Igarashia ${ }^{a}$ Kumie Kodera ${ }^{a}$, Tazuo Okuno ${ }^{a}$, Takuro Morita ${ }^{a}$, Taroh Himeno ${ }^{a}$, \\ Kazu Hamada a, Hiroshi Yano ${ }^{\text {a }}$, Toshihiro Higashikawa ${ }^{\mathrm{a}}$, Osamu Iritani ${ }^{\mathrm{a}}$, Kunimitsu Iwai ${ }^{\mathrm{a}}$, \\ Shigeto Morimoto ${ }^{\mathrm{a}}$, Masashi Okuro ${ }^{\mathrm{a}, \mathrm{b}}$
}

\begin{abstract}
Background: We have reported that hypertension on admission in elderly patients with acute cerebral infarction is an independent predictor for the development of acute pneumonia. However, the relationship between blood pressure on admission owing to cerebral hemorrhage and the development of pneumonia has not been fully investigated. In this study, we evaluated the relationship between blood pressure levels on admission and the development of pneumonia in elderly patients with cerebral hemorrhage who were in the acute phase.
\end{abstract}

Methods: Subjects consisted of 117 elderly patients with cerebral hemorrhage who were in the acute phase and were emergently admitted to the Department of Geriatric Medicine, Kanazawa Medical University between 2005 and 2015 (59 males and 58 females, the mean age \pm standard deviation (SD) of $80 \pm 8$ years, and the range of 65 - 98 years). Blood pressure levels on admission were classified into the following four groups: normal blood pressure/mild hypertension group (systolic blood pressure of $<160 \mathrm{~mm} \mathrm{Hg}$ and diastolic blood pressure of $<100 \mathrm{~mm} \mathrm{Hg}$ ), moderate hypertension group (systolic hypertension of $160-179 \mathrm{~mm} \mathrm{Hg}$ or diastolic blood pressure of $100-109 \mathrm{~mm} \mathrm{Hg}$ ), severe hypertension group (systolic hypertension of $180-199 \mathrm{~mm} \mathrm{Hg}$ or diastolic blood pressure of 110 - $119 \mathrm{mmHg}$ ), and serious hypertension group (systolic blood pressure of $\geq 200 \mathrm{~mm} \mathrm{Hg}$ or diastolic blood pressure of $\geq 120 \mathrm{~mm} \mathrm{Hg}$ ). Between the two groups (group of patients with acute pneumonia and group of those with absence of pneumonia), age, sex, body mass index (BMI), history of stroke, history of heart disease, chronic kidney disease, diabetes, dyslipidemia, prehypertension, blood pressure on admission, Japan Coma Scale (JCS) on admission, white blood cell count, C-reactive protein (CRP), albumin, bleeding sites, bleeding amount, and the presence or absence of centerline shift

Manuscript submitted August 24, 2020, accepted September 22, 2020

Published online November 3, 2020

aDepartment of Geriatric Medicine, Kanazawa Medical University, Uchinada, Kahoku-gun, Ishikawa 920-0293, Japan

${ }^{b}$ Corresponding Author: Masashi Okuro, Department of Geriatric Medicine, Kanazawa Medical University, Uchinada, Kahoku-gun, Ishikawa 920-0293, Japan.Email: okuro@kanazawa-med.ac.jp

doi: https://doi.org/10.14740/jocmr4330 on brain computed tomography (CT) images were retrospectively evaluated. Furthermore, factors related to cerebral hemorrhage in the development of acute pneumonia in patients with cerebral hemorrhage were verified.

Results: Of the 117 patients, 30 (25.6\%) had acute pneumonia. Age, sex, bleeding amount, midline shift, blood pressure classification on admission, JCS, white blood cell count, CRP, albumin, diabetes were adopted as confounding factors in the development of acute pneumonia. Results of multiple logistic regression analysis showed significant differences between these two groups in the following four items: CRP, white blood cell count, JCS, and blood pressure classification on admission. After adjustment of these confounding factors, the incidence of acute pneumonia in the blood pressure groups other than serious hypertension group was set as 1 , and the odds ratio of pneumonia onset in serious hypertension group was revealed to be 5.54 , with the $95 \%$ confidence interval of $1.49-20.6$

Conclusions: We found that serious hypertension on admission is a risk factor for the development of acute pneumonia in elderly patients with cerebral hemorrhage who are in the acute phase.

Keywords: Elderly; Cerebral hemorrhage; Blood pressure on admission; Acute pneumonia

\section{Introduction}

In Japan, cerebral hemorrhage used to account for the majority of strokes, but the proportion has been decreasing due to the spread of antihypertensive therapy and the improvement of dietary habits. However, in recent years, the decrease seems to have reached its limit, and the rate of cerebral hemorrhage is still higher in Japan compared to Europe and the USA [1,2].

Pneumonia is known as one of the diseases most frequently observed as a complication of cerebral hemorrhage in the acute phase $[3,4]$. In addition, patients who have developed pneumonia are also vulnerable to other complications; and pneumonia is a factor that makes condition worsen even 
in relatively mild cerebral hemorrhage with poor prognosis [4]. On the other hand, preventive measures such as early resting deposition/sitting encouragement, early intervention of a speech therapist or speech-language-hearing therapist (ST), and rapid administration of antibiotics in patients with fever have also been shown to reduce the complication rate of pneumonia [3]. Therefore, prediction of pneumonia onset in the acute phase of cerebral hemorrhage is very important.

We have also reported that serious hypertension of elderly patients with acute cerebral infarction on admission is an independent predictor for the development of acute pneumonia [5], and it is expected that the same association will be found for cerebral hemorrhage. However, the number of reports that have shown the association between hypertension on admission and the development of pneumonia in elderly patients who were in the acute phase of cerebral hemorrhage is still quite limited.

In this study, we investigated the relationship between blood pressure on admission and the onset of acute pneumonia in elderly patients with cerebral hemorrhage who were in the acute phase.

\section{Materials and Methods}

\section{Subjects}

Subjects consisted of successive 117 patients who were emergently diagnosed by cerebral computed tomography (CT) with cerebral hemorrhage and were admitted to the Department of Geriatric Medicine, Kanazawa Medical University between 2005 and 2015 (59 males and 58 females, the mean age \pm standard deviation (SD) of $80 \pm 8$ years, and the range of 65 98 years). While patients for whom the cerebral CT scan and blood test were not conducted are excluded, there were no excluded patients during this study.

\section{Methods}

For 117 patients described above, we retrospectively evaluated the clinical survey items which will be described later to compare the differences between the two groups: acute pneumonia group and group of those with the absence of pneumonia (to be described as "non-pneumonia group" hereinafter).

\section{Statistical analysis}

In the univariate analysis of comparing these two groups that had been classified according to the presence or absence of acute pneumonia, we used Mann-Whitney U test for continuous values and $\chi^{2}$ test for categorical variables. Furthermore, we performed multiple logistic regression analysis with age, sex, and all the other factors showing the tendency to involve the $\mathrm{P}$ value of $<0.1$ as the confounding factors in our univariate analysis between the two groups. In multiple logistic regression analysis, we determined it to be statistically significant with $\mathrm{P}$ value of $<0.05$.

\section{Classification of blood pressure levels on admission}

Blood pressure level on admission was defined as the maximum blood pressure which was measured within $72 \mathrm{~h}$ after admission. During this period, vital signs, including noninvasive cuff blood pressure, pulse rate and body temperature, were measured by the nurses at least every $2 \mathrm{~h}$ during the first $24 \mathrm{~h}$ and continued every $2-8 \mathrm{~h}$ up to $72 \mathrm{~h}$. We classified the blood pressure levels into the following four groups by referring to the report of Bath et al and Vaughan et al $[6,7]$. 1) Normal blood pressure/mild hypertension group: systolic blood pressure of less than $160 \mathrm{~mm} \mathrm{Hg}$ and diastolic blood pressure of less than $100 \mathrm{~mm} \mathrm{Hg}$; 2) Moderate hypertension group: systolic blood pressure of $160-179 \mathrm{~mm} \mathrm{Hg}$, or diastolic blood pressure of 100 - 109 mm Hg; 3) Severe hypertension group: systolic blood pressure of 180 - $199 \mathrm{~mm} \mathrm{Hg}$, or diastolic blood pressure of 110 - $119 \mathrm{~mm} \mathrm{Hg}$; 4) Serious hypertension group: systolic blood pressure of $200 \mathrm{~mm} \mathrm{Hg}$ or higher, or diastolic blood pressure of $120 \mathrm{~mm} \mathrm{Hg}$ or higher.

\section{Clinical survey items}

Regarding clinical background, we investigated the age, sex, body mass index (BMI), history of stroke, and history of heart disease. As underlying diseases, we investigated the presence or absence of chronic kidney disease (estimated glomerular filtration rate $(\mathrm{eGFR})<60 \mathrm{~mL} / \mathrm{min}$ ), diabetes (hemoglobin A1c (HbA1c) (National Glycohemoglobin Standardization Program (NGSP)) $>6.5 \%$, or the use of hypoglycemic agents and insulins), dyslipidemia (fasting plasma of low-density lipoprotein cholesterol (LDL-C) $>140 \mathrm{mg} / \mathrm{dL}$, or triglycerides of $>150$ $\mathrm{mg} / \mathrm{dL}$ ), and pre-hypertension (untreated hypertension at the stage before the onset of cerebral hemorrhage, or administration of antihypertensive agent). We investigated blood pressure levels on admission, Japan Coma Scale (JCS) on admission, white blood cell count, C-reactive protein (CRP), serum albumin value, bleeding sites, bleeding amount $(\mathrm{ABC} / 2$ on brain $\mathrm{CT}$ images; $\mathrm{A}=$ maximum diameter, maximum diameter perpendicular to $\mathrm{B}=\mathrm{A}, \mathrm{C}=$ slice width [8]), and midline shift on brain CT images both on admission and after hospitalization.

\section{Diagnostic criteria for objective variables}

Referring to the report by Indredavik et al [9], acute pneumonia was defined as appearance of rale sounds during chest auscultation within 14 days of admission, pyrexia of $37.5^{\circ} \mathrm{C}$ or more, abnormally high value of CRP, increase in peripheral white blood cell count of $9,000 / \mu \mathrm{L}$ or more, appearance of pulmonary infiltrative shadow in chest $\mathrm{CT}$, or viscous sputum, and requiring treatment and antibiotics.

\section{Ethical considerations}

This study has been approved by the Ethics Committee (approval number I-098) at the facility and is carried out in ac- 
cordance with the guidelines of Declaration of Helsinki.

\section{Results}

Of the 117 patients, $30(25.6 \%)$ had acute pneumonia. The group of patients with pneumonia (pneumonia group) showed a tendency of age higher than the non-pneumonia group, but no significant differences were observed in sex, BMI, or bleeding sites. On the other hand, bleeding amount and midline shift were significantly higher in the pneumonia group. In the pneumonia group, moreover, blood pressure levels and JCS on admission were significantly higher than those of the nonpneumonia group. In the test results, white blood cell count and CRP on admission showed high values, but serum albumin levels were significantly lower instead. In the items such as the past medical history and the underlying diseases, the pneumonia group showed a tendency of higher rate of having diabetes complication, but there were no significant differences observed in other items. In the blood pressure before the emergency visits, no significant differences were observed in prehypertension, untreated hypertension, or the use of antihypertensive agents or their types between the pneumonia group and non-pneumonia group (Table 1).

Considering what have been stated so far, age, sex, amount of hemorrhage, midline shift, JCS, white blood cell count, CRP, serum albumin value, and diabetes were taken as the confounding factors for the occurrence of acute pneumonia.

Results of multiple logistic regression analysis showed that CRP, white blood cell count, and JCS as well as blood pressure classification on admission were considered independent significant factors related to the development of acute pneumonia in patients with cerebral hemorrhage (Table 2).

After these confounding factors had been adjusted, the incidence of acute pneumonia in the serious hypertension group was evaluated. When the rate of acute pneumonia onset was set as 1 for other blood pressure groups, the odds ratio of pneumonia onset in the serious hypertension group was 5.54, with the $95 \%$ confidence interval of 1.49 - 20.6 (Table 3 ).

\section{Discussion}

In this study, acute pneumonia was noted in $25.6 \%$ of the subjects. Pneumonia is said to develop in $21.1-26.9 \%$ of the patients with cerebral hemorrhage who are in the acute phase $[4$, $10]$, and the study revealed similar results in terms of incidence of acute pneumonia as have been reported in previous studies.

In this study, the group who presented with acute pneumonia showed a tendency to have significantly higher blood pressure levels on admission, and the classification of blood pressure levels on admission was considered to be one of the independent and significant related factors for the development of pneumonia. On the other hand, no significant differences were noted in the presence or absence of hypertension before the onset of cerebral hemorrhage or the types of antihypertensives between the pneumonia group and non-pneumonia group. The result suggests that a group in which the blood pressure rapidly increases following the onset of cerebral hemorrhage actually exists, and the group is more vulnerable to develop pneumonia. It is unlikely that increased blood pressure is directly related to the development of pneumonia, and it is considered that there are some other factors involved in both increased blood pressure and the development of pneumonia in patients with cerebral hemorrhage.

Increased catecholamine occurs at the onset of cerebral hemorrhage, and is known to be one of the conditions that can explain the involvement of both increased blood pressure and the onset of pneumonia. In the acute phase of cerebral hemorrhage and cerebral infarction, adrenaline and noradrenaline concentrations in the blood and cerebrospinal fluid are known to increase due to the sympathetic nervous system being overactive [11]. Moreover, a report indicates that immunity decreased due to overactivity of the sympathetic nervous system in the stroke model using mice $[12,13]$. Overactivity of the sympathetic nervous system triggered by the onset of cerebral hemorrhage induces increased catecholamine concentration, leading to increased blood pressure, followed by the patients becoming even more vulnerable to develop pneumonia, because their immunity is decreased.

In addition, gene polymorphism such as angiotensin-converting enzyme (ACE) is also considered to be involved. ACE is an enzyme that produces angiotensin II that has a strong vasopressor effect. Angiotensin I is a substrate of angiotensin II. It is considered likely that, in patients in whom ACE concentration is high, blood pressure is likely to increase more easily [14-17].

In addition, ACE is also responsible for decomposing bradykinin and substance $\mathrm{P}$, both of which are important for cough reflex and swallowing reflex; and it has also been reported that I/D polymorphism may be associated with cough reflex [18]. In our previous studies, patients with ACE-DD polymorphism showed significantly more number of pneumonia onset and death caused by pneumonia, as compared with patients with ACE-ID polymorphism or ACE-II polymorphism. It is considered that ACE D allele reduces local concentrations of bradykinin and substance P by increased ACE activity in the blood and tissues, leading to decreased cough reflex and the patients becoming more vulnerable to the onset of pneumonia [19].

Furthermore, we have previously reported the relationship between acute cerebral infarction, blood pressure, and pneumonia in the elderly [5], and the results of this study suggest that the same association is found in patients with cerebral hemorrhage. We consider that the relationship between blood pressure on admission and the onset of pneumonia is a matter commonly observed in patients with stroke in general. However, in this study, it is difficult to sufficiently verify whether the backgrounds and disease images of pneumonia in patients with cerebral infarction/cerebral hemorrhage are the same, and the accumulation of future findings is awaited.

This study suggested the relationship between blood pressure and the onset of pneumonia in patients with the acute phase of cerebral hemorrhage, but it is unknown whether antihypertensive treatment in the acute phase leads to the prevention of pneumonia onset. As mentioned above, if some factors generated from cerebral hemorrhage were involved in both increased blood pressure and the onset of pneumonia, solo hypotensive treatment after hospitalization may not pre- 
Table 1. Clinical Background at the Time of Hospitalization

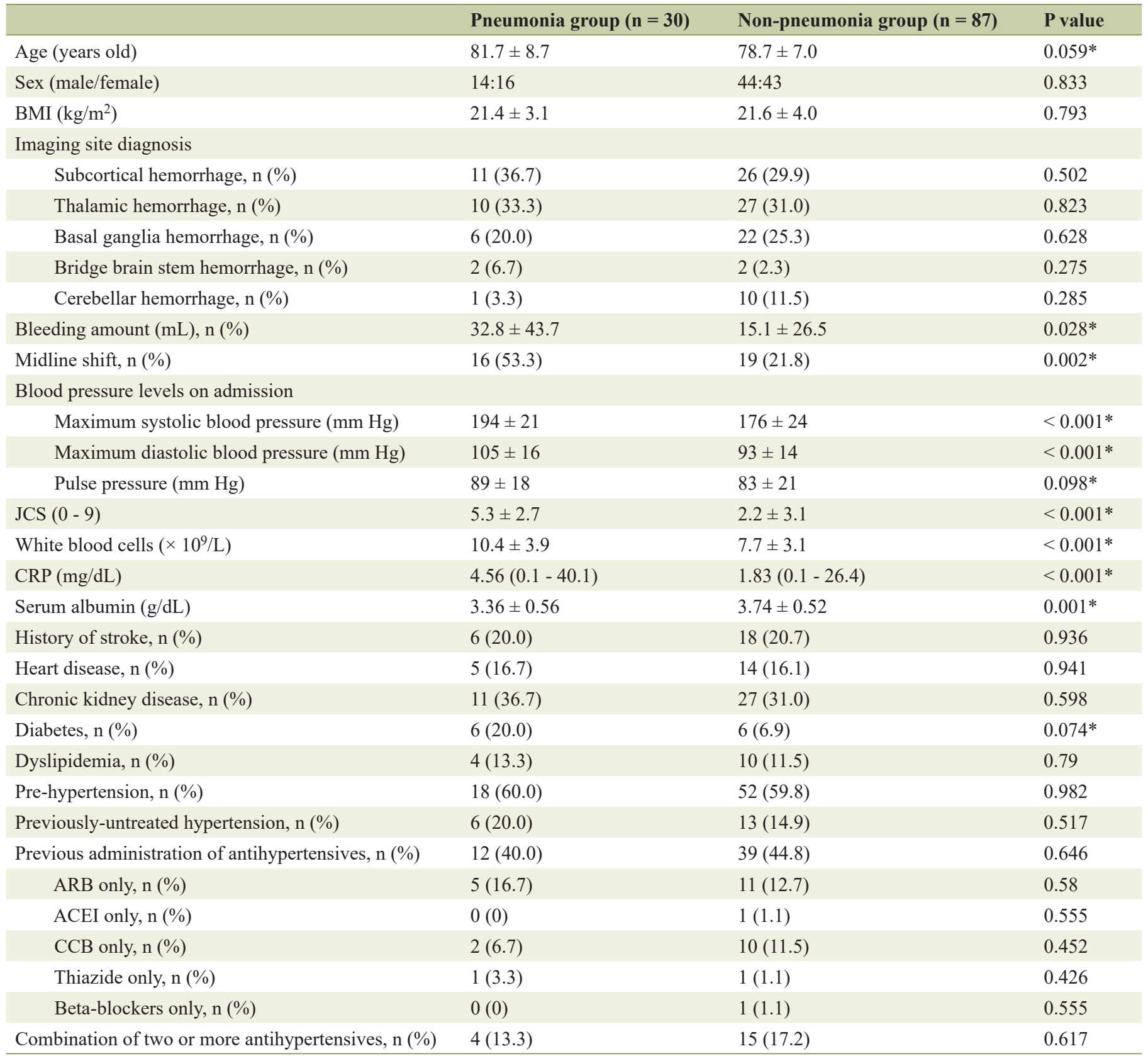

Mann-Whitney $\mathrm{U}$ test, $\mathrm{X}^{2}$ test. ${ }^{*} \mathrm{P}<0.1$. BMI: body mass index; JCS: Japan Coma Scale; CRP: C-reactive protein; ARB: angiotensin II receptor blocker; ACEl: angiotensin-converting enzyme inhibitor; CCB: calcium channel blocker.

vent the onset of pneumonia. Moreover, from the viewpoint of dysfunction and safety, the Japanese guidelines have already recommended to reduce systolic blood pressure to $<140 \mathrm{~mm}$ $\mathrm{Hg}$ for patients with cerebral hemorrhage [20]. Even if antihypertensive treatment is useful for the prevention of pneumonia, the results of this study indicate that antihypertensive treatment above the current guidelines is not recommended. As the preventive measures of pneumonia associated with stroke, early resting deposition, ST intervention, rapid administration of antibiotics at the onset of fever, and administration of ACE inhibitors and cilostazole are considered to be useful $[3,21$, 22]. If blood pressure levels on admission are high in patients with cerebral hemorrhage, especially in the patients presenting serious hypertension, they are considered a high-risk group of pneumonia onset. It is considered desirable to take these preventive measures for pneumonia onset aggressively and intensively in such patients.

Further, microbiological examination of tracheal specimens and/or blood cultures did not detected atypical pathogens, such as Chlamydia pneumoniae, Mycoplasma pneumoniae or 
Table 2. Independent Risk Factors for Acute Pneumonia

\begin{tabular}{lllll} 
& Ward & Odds ratio & 95\% confidence interval & P value \\
\hline $\log (\mathrm{CRP})(\mathrm{mg} / \mathrm{dL})$ & 6.96 & 3.23 & $1.35-7.72$ & $0.008^{*}$ \\
Blood pressure on admission 4 classifications & 5.94 & 2.4 & $1.19-4.85$ & $0.015^{*}$ \\
White blood cells $\left(\times 10^{9} / \mathrm{L}\right)$ & 4.3 & 1.21 & $1.01-1.45$ & $0.038^{*}$ \\
JCS $(0-9)$ & 4.27 & 1.3 & $1.01-1.66$ & $0.039^{*}$ \\
Diabetes & 2.72 & 4.12 & $0.77-22.2$ & 0.099 \\
Age $(1$ year old) & 2.23 & 1.07 & $0.98-1.18$ & 0.135 \\
Serum albumin $(\mathrm{g} / \mathrm{dL})$ & 2.19 & 0.4 & $0.12-1.35$ & 0.139 \\
Bleeding amount $(\mathrm{mL})$ & 0.81 & 0.99 & $0.97-1.01$ & 0.368 \\
Midline shift & 0.65 & 0.53 & $0.12-2.48$ & 0.421 \\
Men & 0.44 & 1.53 & $0.43-5.45$ & 0.509 \\
\hline
\end{tabular}

${ }^{*}$ Multiple logistic regression analysis, P < 0.05. CRP: C-reactive protein; JCS: Japan Coma Scale.

Table 3. Relationship Between Acute Pneumonia and Various Blood Pressure Groups on Admission

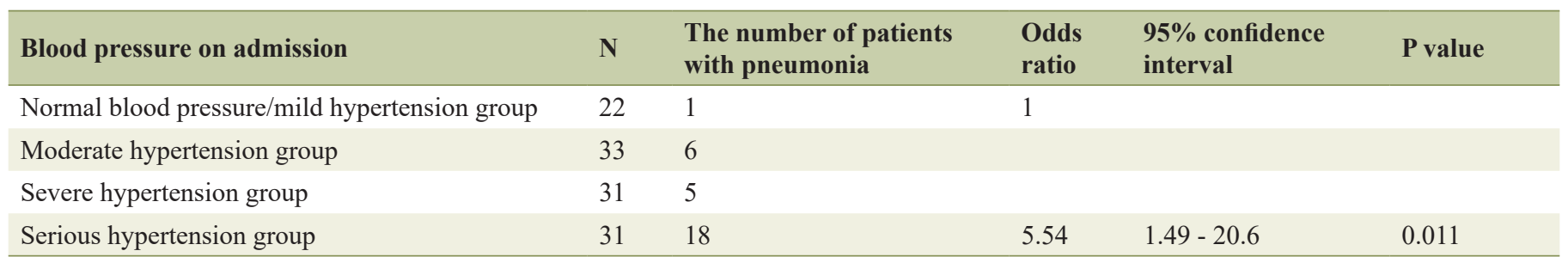

Multiple logistic regression analysis adjusted by age, sex, bleeding amount, midline shift, JCS, white blood cell count, log (CRP), serum albumin, and diabetes. CRP: C-reactive protein; JCS: Japan Coma Scale.

Legionella pneumophila (data not shown). Research including the pathogen is required in order to clarify the mechanism of serious hypertension.

\section{Acknowledgments}

We thank all the study investigators and staff and participants who participated in this study, for helpful discussions during manuscript development.

\section{Financial Disclosure}

None to declare.

\section{Conflict of Interest}

The authors declare no conflict of interest.

\section{Informed Consent}

Patients were not required to give informed consent to the study because the retrospective analysis used anonymous clinical data that were obtained after each patient agreed to the treatment schedule by written consent.

\section{Author Contributions}

Taishi Ishisaka wrote the manuscript; Masashi Okuro and Yuta Igarashi collected data for review; all authors read and approved the final version of the manuscript.

\section{Data Availability}

The data supporting the findings of this study are available from the corresponding author upon reasonable request.

\section{References}

1. Kowa H, Nakajim K. Current status of cerebral hemorrhage in the elderly. Journal of the Japanese Geriatrics Society. 2017;54:514-518.

2. Krishnamurthi RV, Feigin VL, Forouzanfar MH, Mensah GA, Connor M, Bennett DA, Moran AE, et al. Global and regional burden of first-ever ischaemic and haemorrhagic stroke during 1990-2010: findings from the Global Burden of Disease Study 2010. Lancet Glob Health. 2013;1(5):e259-281.

3. Suzuki K, Matsumura A. Systemic complications and countermeasures in the acute phase of cerebral hemorrhage. ICU and CCU. 2008;32:467-474.

4. Yasuda M, Yanaka K, Suzuki K, Matsumura A, Kujiraoka 
Y, Kimura H, Komatsu Y, et al. Systemic complications in patients with cerebral hemorrhage - especially pneumonia. Stroke Surgery. 2003;31:406-409.

5. Ishigami K, Okuro M, Koizumi Y, Satoh K, Iritani O, Yano H, Higashikawa T, et al. Association of severe hypertension with pneumonia in elderly patients with acute ischemic stroke. Hypertens Res. 2012;35(6):648-653.

6. Bath P, Chalmers J, Powers W, Beilin L, Davis S, Lenfant C, Mancia G, et al. International Society of Hypertension (ISH): statement on the management of blood pressure in acute stroke. J Hypertens. 2003;21(4):665-672.

7. Vaughan CJ, Delanty N. Hypertensive emergencies. Lancet. 2000;356(9227):411-417.

8. Kothari RU, Brott T, Broderick JP, Barsan WG, Sauerbeck LR, Zuccarello M, Khoury J. The ABCs of measuring intracerebral hemorrhage volumes. Stroke. 1996;27(8):1304-1305.

9. Indredavik B, Rohweder G, Naalsund E, Lydersen S. Medical complications in a comprehensive stroke unit and an early supported discharge service. Stroke. 2008;39(2):414-420.

10. Harada S, Kumon N, Fukuda S, Uratani A, Funakoshi K, Umai T, Ono A. Formulation of the prediction model for the development of aspiration pneumonia in patients with acute cerebral stroke. Annual report of Kurashiki Central Hospital. 2017;79:65-73.

11. Oto J, Suzue A, Inui D, Fukuta Y, Hosotsubo K, Torii M, Nagahiro S, et al. Plasma proinflammatory and antiinflammatory cytokine and catecholamine concentrations as predictors of neurological outcome in acute stroke patients. J Anesth. 2008;22(3):207-212.

12. Mracsko E, Stegemann-Koniszewski S, Na SY, Dalpke A, Bruder D, Lasitschka F, Veltkamp R. A mouse model of post-stroke pneumonia induced by intra-tracheal inoculation with streptococcus pneumoniae. Cerebrovasc Dis. 2017;43(3-4):99-109.

13. Prass K, Braun JS, Dirnagl U, Meisel C, Meisel A. Stroke propagates bacterial aspiration to pneumonia in a model of cerebral ischemia. Stroke. 2006;37(10):2607-2612.
14. Rice GI, Jones AL, Grant PJ, Carter AM, Turner AJ, Hooper NM. Circulating activities of angiotensin-converting enzyme, its homolog, angiotensin-converting enzyme 2, and neprilysin in a family study. Hypertension. 2006;48(5):914-920.

15. Danser AH, Schalekamp MA, Bax WA, van den Brink AM, Saxena PR, Riegger GA, Schunkert H. Angiotensin-converting enzyme in the human heart. Effect of the deletion/insertion polymorphism. Circulation. 1995;92(6):1387-1388.

16. O'Donnell CJ, Lindpaintner K, Larson MG, Rao VS, Ordovas JM, Schaefer EJ, Myers RH, et al. Evidence for association and genetic linkage of the angiotensin-converting enzyme locus with hypertension and blood pressure in men but not women in the Framingham Heart Study. Circulation. 1998;97(18):1766-1772.

17. Higaki J, Baba S, Katsuya T, Sato N, Ishikawa K, Mannami T, Ogata $J$, et al. Deletion allele of angiotensinconverting enzyme gene increases risk of essential hypertension in Japanese men : the Suita Study. Circulation. 2000;101(17):2060-2065.

18. Li YF, Zhu XM, Liu F, Xiao CS, Bian YF, Li H, Cai J, et al. Angiotensin-converting enzyme (ACE) gene insertion/deletion polymorphism and ACE inhibitor-related cough: a meta-analysis. PLoS One. 2012;7(6):e37396.

19. Morimoto S, Okaishi K, Onishi M, Katsuya T, Yang J, Okuro M, Sakurai S, et al. Deletion allele of the angiotensin-converting enzyme gene as a risk factor for pneumonia in elderly patients. Am J Med. 2002;112(2):89-94.

20. Ogawa A. Treatment of hypertensive cerebral hemorrhage of the acute phase management of blood pressure: guidelines for stroke treatment. The Japan Stroke Society. 2015;2015:143-144.

21. Mukamal KJ, Ghimire S, Pandey R, O'Meara ES, Gautam $\mathrm{S}$. Antihypertensive medications and risk of communityacquired pneumonia. J Hypertens. 2010;28(2):401-405.

22. Osawa A, Maeshima S, Tanahashi N. Efficacy of cilostazol in preventing aspiration pneumonia in acute cerebral infarction. J Stroke Cerebrovasc Dis. 2013;22(6):857-861. 